\title{
Equidad de Género en el Acceso a los Servicios de Salud en Colombia
}

\author{
Gender-related equitylinequity in gaining access to health services \\ Carol C. Guarnizo-Herreño ${ }^{1}$ y Carlos Agudelo ${ }^{2}$
}

1 Facultad de Odontología, Universidad Nacional de Colombia, Bogotá. ccguarnizoh@unal.edu.co 2 Instituto de Salud Pública, Universidad Nacional de Colombia. Bogotá. caagudeloc@unal.edu.co

Recibido 8 Enero 2007/Enviado para Modificación 2 Mayo 2008/Aceptado 3 Septiembre 2008

\section{RESUMEN}

Objetivo Identificar inequidades de género en el acceso a los servicios de salud en el sistema de seguridad social en salud.

Métodos Se realizó una evaluación de dos etapas de la Encuesta Nacional de Hogares, antes y después de implementada la reforma al sistema de salud colombiano. Se identificaron variables socioeconómicas, de necesidad, acceso a los servicios y financiación. Se realizó un análisis descriptivo de las variables, se realizó la recodificación y creación de nuevas variables. Se hicieron análisis bivariados, comparando algunas variables por medio de $\mathrm{Chi}^{2}$.

Resultados La necesidad en salud sentida fue mayor en las mujeres en las dos encuestas. Las diferencias entre hombres y mujeres en la consulta por problema de salud no fueron significativas en 1994; en el 2000, consultaron el $73 \%$ de los hombres y el $81 \%$ de las mujeres $(p<0.0001)$. Respecto al antecedente de hospitalización, la proporción de hombres y mujeres que utilizaron este servicio disminuyó en el 2000. En el 2000, las mujeres emplearon en mayor proporción los recursos propios como fuente de financiación. Por niveles de ingreso, las personas pertenecientes a los quintiles uno y dos emplearon los recursos propios como fuente de financiación de los servicios.

Conclusiones Al comparar los dos períodos, se incrementó el total de consultas, más rápidamente en las mujeres. En el año 2000 las mujeres tuvieron más acceso al servicio de consulta. Las inequidades en el financiamiento de los servicios de salud por sexo han persistido aún después de implementada la reforma.

Palabras Clave: Equidad en salud, equidad en el acceso, género, financiación, acceso a los servicios de salud (fuente: DeCS, BIREME).

\section{ABSTRACT}

Objective Identifying gender-related inequities in gaining access to health services in the Colombian social health security system.

Methods A two-stage evaluation of the National Household Survey was made, before and after the Colombian health system reform was implemented. Socioeconomic, 
needs, access to health services and financing variables were identified. A descriptive analysis of the variables was made, they were re-codified and new variables created. Bivariate analysis was done, comparing some variables by means of Chi².

Results Needs in health were felt more by women in both surveys. Differences between males and females in consultation for health problems were not significant in 1994; $73 \%$ of the males and $81 \%$ of the females were consulted in $2000(p<0.0001)$. Regarding antecedents of having been hospitalised, the percentage of males and females using this service became reduced during 2000. Females used their own resources more as the source of financing services in 2000. People belonging to the $1^{\text {st }}$ and $2^{\text {nd }}$ quintiles used their own resources as financing source in terms of income levels.

Conclusions Comparing both periods, total consultations became increased (more rapidly in females). Females had greater access to consultation services in 2000. Gender-related inequities in financing health services have still persisted, even after the reform was implemented.

Key Words: Equity in health, equity in access, gender, financing, access to health services (source: $\mathrm{MeSH}, \mathrm{NLM}$ ).

l aumento de los niveles de desigualdad en los últimos años en América
Latina y el Caribe ha hecho que el debate político y académico sobre
equidad haya aumentado de manera importante; en salud específicamente las reflexiones frente a la equidad incluyen debates que, inspirados en distintas corrientes políticas y filosóficas, asumen una postura acerca de lo que se considera injusto en el campo sanitario, así como ejercicios de evaluación de las políticas implementadas en los países en términos de superación de las inequidades existentes. En la región, durante la década de los noventa se implementaron en la mayoría de países reformas al sector salud en el marco de procesos de ajuste macroeconómico y de reforma del Estado. Estas reformas tenían entre sus objetivos principales el incremento de la equidad en acceso a servicios, sin embargo, luego de varios años de su implementación, persisten desigualdades en el estado de salud, en la oferta de servicios, acceso y financiamiento; estas diferencias se presentan entre los países, entre regiones al interior de los países y entre distintos grupos sociales definidos por nivel socioeconómico, etnia, género y edad (1).

En Colombia, la reforma al sistema de salud cristalizada en la ley 100 de 1993 se planteó garantizar la equidad en términos de acceso, utilización y financiamiento de los servicios de salud; considerando que han transcurrido varios años de su implementación, es necesario evaluar el impacto que en este sentido se ha producido. Aún el sistema no alcanza cobertura universal y los mayores rezagos recaen sobre los más pobres, en los cuales la afiliación es 
menor; estas diferencias aumentan cuando el criterio anterior es analizado en función de la zona de residencia, resultando la zona rural como la más afectada (2). Un factor adicional que genera inequidad en el acceso a los servicios en detrimento de los más pobres es el derivado de las diferencias y limitaciones entre los planes de beneficio de los regímenes, lo que ha promovido un mayor gasto de bolsillo en la población más pobre (3)

De otra parte, distintos estudios señalan a la condición de género como otro de los factores que explican inequidades en salud. De hecho uno de los aspectos mas importantes que determina los procesos de acceso, utilización y financiamiento de los servicios es el género, entendido como la construcción social de roles a partir de características biológicas. La evaluación de la equidad de género en el acceso a servicios de salud es un aspecto que aún no se ha estudiado lo suficiente en nuestro país y que merece un análisis juicioso desde distintas disciplinas. El presente estudio se propuso identificar diferencias de género en el acceso y el financiamiento de los servicios de salud en el Sistema General de Seguridad Social en Salud, a partir de información de dos etapas de la Encuesta Nacional de Hogares (ENH), en 1994 -cuando aún no se había implementado la reforma al sistema de salud- y el 2000, seis años después de su implementación.

\section{Referentes conceptuales}

En el presente trabajo se asume la inequidad en salud como la presencia de desigualdades innecesarias, injustas y evitables en los fenómenos de salud, enfermedad y muerte entre poblaciones o grupos poblacionales definidos social, económica, demográfica o geográficamente; en esta perspectiva las inequidades se producen cuando por condiciones de ingreso, raza, sexo, edad, religión u otras situaciones que no han sido producto de una elección voluntaria, los individuos o los colectivos no logran tener las mismas oportunidades para gozar de buena salud (4). En términos de capacidades, se podría decir que idealmente todas las personas tendrían la oportunidad efectiva de alcanzar su máximo potencial en salud y que ninguna persona debería estar en desventaja para alcanzar este potencial (5). Partiendo de la valoración de la salud como un derecho fundamental, todos aquellos factores que se constituyan en barreras para su logro y que sean modificables deberán considerarse como objeto de trabajo desde la política pública y responsabilidad del Estado. 
Estos conceptos han sido desarrollados ampliamente por un grupo de investigadores entre los que se destaca Margaret Whitehead para quien es importante diferenciar las desigualdades consideradas injustas y evitables de aquellas que no lo son. Es importante considerar en los análisis de equidad aquellas diferencias que afectan a ciertas personas por condiciones que ellas no han elegido; estas condiciones en muchos casos se refuerzan unas a otras haciendo a algunos grupos más vulnerables frente a las condiciones de salud. El enfoque de exclusión social ayuda en este tópico al permitir el análisis de los cruces y relaciones de las distintas formas de carencia. Distintos aspectos de la privación se acumulan, por ejemplo, la falta de ingreso está relacionada con desempleo o formas precarias del empleo, pero también con mala salud física o mental, falta de vivienda adecuada, falta de educación o mala calidad en la educación recibida, mala alimentación y exclusiones relacionadas con condiciones género. De lo anterior se deduce que los grupos que sufren privaciones generalmente soportan desventajas múltiples y acumulativas (6).

\section{Equidad en los servicios de salud}

Basados en las anteriores definiciones es posible afirmar que las políticas en salud que busquen equidad tendrán como objetivo reducir o eliminar aquellas diferencias que resultan de factores considerados evitables e injustos; creando igualdad de oportunidades para alcanzar el estado de salud deseable. En general, se considera que existe equidad en los servicios de salud cuando se cumplen las siguientes condiciones: los recursos son asignados de acuerdo a la necesidad, los servicios de salud se reciben de acuerdo a la necesidad y el pago es hecho de acuerdo a la capacidad. A partir de estas definiciones, la equidad en la prestación de servicios seguiría las premisas: igual acceso a los servicios de salud para igual necesidad, igual uso de servicios para igual necesidad e igual calidad de atención para todos.

Acceso. En arreglo a las anteriores consideraciones, las inequidades en el acceso se producen cuando por condiciones de ingreso, raza, sexo, edad, religión y otros factores, los individuos no logran utilizar los servicios de salud, esto ocurrirá también cuando los recursos y facilidades están desigualmente distribuidos en el territorio nacional, por ejemplo cuando estos se encuentran concentrados en las áreas urbanas.

Travassos y cols (7) hacen claridad en la diferencia entre equidad en salud y equidad en la utilización de los servicios de salud; llamando la atención en que no todos los aspectos determinantes de las desigualdades en los indicadores de 
salud intervienen en la determinación de las desigualdades en el uso de los servicios de salud.

Teniendo en cuenta que la afirmación esbozada sobre lo que se consideran son los servicios de salud equitativos parte del referente de necesidad, es pertinente discutir con mayor atención este concepto y su cadena de relaciones. Consideremos la siguiente situación: un individuo siente que está enfermo y a partir de esa percepción identifica que necesita atención en salud; estas sensaciones se encuentran mediadas por aspectos culturales y sociales (8) que determinan en principio que esta persona piense en los servicios de salud como mecanismo de solución a su problema. A partir de la necesidad, el individuo efectivamente busca la atención. En esta búsqueda ocurren una serie de fenómenos que tienen relación tanto con la disposición oportuna y suficiente del servicio como con aspectos de su vida social y colectiva, lo que en términos de lo expuesto, se constituye en el proceso de acceso a los servicios del sistema de salud. Ahora bien, si este proceso es exitoso se llega entonces a la utilización efectiva de los servicios. Es en consecuencia la necesidad percibida desde donde se desencadenan el conjunto de sucesos que pueden resultar o no en el uso efectivo del servicio y son a su vez estos elementos los que deben ser sometidos a una valoración de justicia. A este respecto se afirma que las necesidades en salud están socialmente determinadas y que a su vez el uso de los servicios de salud está determinado por las necesidades de salud de la población y por las características de la oferta del mercado de servicios de salud (política de salud)(7).

Financiamiento. En términos del financiamiento en salud, se consideran los gastos efectuados a través de impuestos directos e indirectos, aportes a seguridad social, seguros privados y gastos de bolsillo. Desde un enfoque igualitarista, la equidad financiera de un sistema de salud se logrará cuando cada individuo u hogar contribuya a la recolección de los fondos según su capacidad de pago y ninguna otra variable como sexo, raza, lugar de residencia, estado civil, estado de salud, entre otras, determine el aporte que realice. Para que esto sea posible se debe garantizar que los más pobres paguen relativamente menos en comparación con su renta y los más ricos relativamente más. Lo anterior para que se cumpla el objetivo de que la financiación del sistema de salud no empeore la distribución del ingreso ya existente.

Teniendo en cuenta que los sistemas de salud se financian por distintas fuentes, la regresividad/progresividad de todo el sistema dependerá de las características de estos esquemas de recolección de recursos, los cuales se pueden agrupar en: 
impuestos directos, impuestos indirectos, impuestos a la nómina, seguros privados y gasto de bolsillo, esta última es considerada una fuente muy regresiva pues pagarán aquellas personas que consultan o se enferman, lo cual recaerá más duramente sobre el ingreso de los hogares más pobres, este gasto privado es como porcentaje mayor para los hogares de rentas bajas (9).

Equidad, género y salud. La categoría sexo hace referencia a las características biológicas que diferencian a hombres y mujeres. A partir de estas diferencias se construye un significado social: el género, basado en la división según el sexo, de los roles y el poder; de esta manera el género se convierte en uno de los ejes primarios alrededor de los cuales se organiza la vida social (10). En palabras de Gómez (11), la equidad de género en salud consistirá en la eliminación de aquellas desigualdades evitables en las oportunidades de disfrutar de la salud y de no enfermar, discapacitarse o morir por causas prevenibles. Las inequidades de género en salud abarcan las siguientes dimensiones:

- Estado de salud y sus determinantes

- Acceso efectivo a atención de acuerdo con necesidad

- Financiamiento de la atención según capacidad de pago

- Balance en la distribución de la carga de responsabilidades y poder en el cuidado de la salud.

La equidad de género en el acceso a los servicios de salud se logrará cuando: se asignen los recursos de atención según las necesidades de hombres y mujeres; los servicios se reciban de acuerdo con las necesidades de cada sexo y los servicios se paguen según la capacidad económica de las personas, no según la necesidad o riesgo diferencial por sexo. Al introducir la perspectiva de género en el análisis de acceso a los servicios de salud, se intenta valorar el impacto de la división sexual del trabajo y el acceso diferencial a los recursos que hacen posible el pago directo o el aseguramiento público o privado.

Aunque las mujeres consumen más servicios de salud que los hombres, esto no significa que exista un «privilegio social» a favor de ellas en este aspecto. Se plantean tres principales razones que explican este patrón diferencial: 1distintos tipos de necesidades de atención; diferentes patrones de socialización con relación al reconocimiento de síntomas, la percepción de la enfermedad y la conducta para buscar atención; y los factores estructurales e institucionales que facilitan u obstruyen diferencialmente el acceso a los servicios de salud. 
Las necesidades de servicios de salud son diferentes para hombres y mujeres por las características biológicas propias de cada sexo: en las mujeres las necesidades incluyen además de las que comparten con los hombres todas las relacionadas con las funciones reproductivas; adicionalmente ellas presentan más altas tasas de morbilidad y discapacidad, y debido a su mayor longevidad tienen mayores riesgos de sufrir enfermedades crónicas. En las mujeres la autopercepción de la enfermedad es más frecuente que en los hombres, la cual se relaciona con las distintas características de socialización, pues en el ejercicio del rol de «cuidadoras de la salud» las mujeres se hacen más hábiles en la detección de síntomas de dolencias y en los procesos formales o informales de cuidado de la salud; mientras aún persiste entre los hombres la percepción de enfermedad y búsqueda de atención como evidencia de debilidad que va en contra de la construcción social de lo masculino como fortaleza, valentía, autosuficiencia y dominio (12). Frente a los factores relacionados con características de los servicios de salud que favorezcan a uno u otro sexo, Gómez afirma que un sistema de salud puede inducir una mayor utilización de servicios por las mujeres gracias a la "medicalización de procesos biológicos", el subsidio a servicios como anticonceptivos y la fragmentación de la atención; adicionalmente sostiene que al utilizar más los servicios preventivos, las mujeres estarán más capacitadas para detectar síntomas que requieran posterior utilización de servicios.

En los sistemas de salud en los cuales el financiamiento no obedece al principio de equidad ya señalado, las barreras estructurales de acceso para las mujeres se dan porque ellas requieren más servicios de salud y en caso de ser estos financiados con contribuciones de bolsillo o por aseguramiento vía vinculación laboral formal, las mujeres incurrirán en mayores gastos que los hombres para mantener su salud; adicionalmente las condiciones laborales y de capacidad económica que tienen las mujeres como grupo las colocan en mayores desventajas respecto a los hombres en términos de acceso a los servicios de salud en sistemas de financiamiento no solidario. Las nuevas dinámicas económicas y políticas mundiales afectan negativamente a las mujeres que viven del trabajo debido a los recortes en los programas sociales, la precarización del trabajo (en calidad, inestabilidad y bajos ingresos) y la nuevas formas de contratación (maquilas y subcontratación para desarrollar el trabajo en el hogar) conduciéndolas a asumir nuevos roles, amortiguar en el hogar el desplome de la calidad de vida, soportar una mayor explotación e inestabilidad en el trabajo y a recortar sus espacios de socialización y acción política (13). 


\section{METODOLOGÍA}

El presente estudio hace parte del proyecto de investigación: “Género y equidad en el acceso a la atención en salud en la reforma a la seguridad social” cuyo objetivo es identificar, con la información actualmente disponible, los patrones, tendencias, determinantes y consecuencias de la inequidad según género en la salud y la atención en salud. En este caso se empleó como fuente de información la Encuesta Nacional de Hogares (ENH), de los años 1994 y 2000, en la cual se identificaron algunas variables proxy de la categoría género: sexo, edad, estado conyugal e ingreso, estas variables tienen limitaciones porque no permiten una identificación más precisa de las construcciones sociales. Dentro de las opciones de medición de las desigualdades en salud se empleó la comparación directa entre grupos, teniendo en cuenta las características de las variables disponibles en las encuestas.

La ENH realizada por el Departamento Administrativo Nacional de Estadística de Colombia (DANE) utiliza muestras aleatorias de la población general e indaga regularmente por características de la población (sexo, edad, estado civil); variables educativas (alfabetismo, asistencia escolar); variables relacionadas con empleo e ingresos y ocasionalmente las ENH han estudiado con mayor detalle aspectos específicos sobre fuerza de trabajo, salud, vivienda y otros.

Para comparar las dos etapas se siguieron los siguientes pasos:

- Identificación de la estructura

- Identificación de las variables, formulación y categorías

- Transformación de las variables

Se evalúo la compatibilidad de las encuestas, se eligieron las variables pertinentes, se obtuvieron frecuencias y se establecieron relaciones entre variables de necesidad, acceso y financiamiento con variables de sexo, edad, estado conyugal e ingreso. Las diferencias entre las categorías establecidas fueron sometidas a un análisis estadístico para valorar su significancia empleando para ello la prueba de Chi cuadrado; esto permitió identificar algunas diferencias consideradas inequidades en los dos períodos, antes y después de implementada la reforma. 


\section{RESULTADOS}

El primer aspecto analizado fue la necesidad en salud sentida o reportada que fue mayor para las mujeres en las dos encuestas analizadas, las diferencias fueron significativas lo cual confirma que existe una diferencia en la necesidad en salud entre hombres y mujeres.

En cuanto a la variable de consulta por problema de salud las diferencias entre hombres y mujeres no fueron significativas en la ENH de 1994; en cambio, en el 2000 las diferencias entre hombres y mujeres en este aspecto se ampliaron, consultaron el $73 \%$ de los hombres y el $81 \%$ de las mujeres $(p<0.0001)$. Teniendo en cuenta que en la ENH del año 2000 se utilizó únicamente la consulta médica, podría afirmarse que se incrementó el total de consultas, pero que se incrementó más rápidamente para las mujeres, es decir que para el año 2000 las mujeres tuvieron más acceso al servicio de consulta.

Respecto al antecedente de hospitalización, los porcentajes de hombres y mujeres que utilizaron este servicio disminuyeron en el 2000. En 1994 reportaron antecedente de hospitalización el 4,7 \% de los hombres y en el 2000 lo hicieron el 3,8 \%; para las mujeres la proporción pasó de $6 \%$ a 5,5 \%; indicando probablemente que el acceso a este tipo de servicios ha disminuido con la reforma para ambos sexos, en los dos períodos las diferencias por sexo fueron significativas con un mayor porcentaje de utilización para las mujeres. Lo anterior responde seguramente a las características de mayor longevidad, mayor morbilidad, más altas tasas de incapacidad y las funciones reproductivas que caracterizan a las mujeres.

Los resultados mencionados hasta el momento se resumen en la tabla 1.

Tabla 1. Necesidad sentida en salud, consulta, hospitalización y vinculación a seguridad social, por sexo 1994 y 2000

\begin{tabular}{lcccc}
\hline & \multicolumn{3}{c}{1984} & \multicolumn{2}{c}{2000} \\
\cline { 2 - 5 } & \% Mujeres & $\%$ Hombres & \% Mujeres & $\%$ Hombres \\
\hline Necesidad sentida & 14,7 & 11,4 & 14,5 & 11,7 \\
Consultaron ante necesidad & 78 & 76 & 81 & 73 \\
Hospitalización último año & 6,1 & 4,7 & 5,6 & 3,8 \\
Seguridad social y seguros & 50 & 54 & & 57 \\
privados & & & 58 & 57 \\
Aseguramiento R. Contributivo & & & & \\
\hline
\end{tabular}


La evaluación en términos de equidad requiere complementar la información respecto al acceso a los servicios con la relativa a la financiación de los mismos. La encuesta incluyó esta variable para el año 2000, evidenciándose que las mujeres emplearon en mayor proporción que los hombres los «recursos propios» como fuente de financiación para su última atención en salud (28\% vs. 25\%, p< 0.0001); de la misma manera la posibilidad que una aseguradora o medicina prepagada sea la fuente de financiación es mayor para los hombres (75 \% vs. 72 $\%, \mathrm{p}<0.0001)$; lo cual coincide con las desventajas que en términos de condiciones laborales tienen las mujeres en Colombia. Estos resultados muestran inequidades en el financiamiento de los servicios de salud por sexo después de implementada la reforma; lo que sugiere que las mujeres se encuentran en situación de desventaja pues los gastos derivados de la atención en salud podrían significar un empobrecimiento para ellas. Teniendo en cuenta que las mujeres se encuentran en mayor porcentaje que los hombres en los quintiles de menor ingreso, el riesgo de empeorar su situación socioeconómica por los gastos de bolsillo empleados en la atención en salud podría ser aún mayor.

En términos de acceso a consulta médica por grupos de edad, en 1994 las diferencias no fueron significativas, en el 2000 las diferencias resultaron significativas con una menor probabilidad de acceder a los servicios en las personas entre 9 y 20 años de edad. Para este año podría afirmarse que las inequidades en el financiamiento, expresadas en el mayor peso del gasto de bolsillo afectaron a los menores de 9 años -a pesar de la introducción del grupo familiar en el aseguramiento-y a las personas entre 15 y 20 años. En estos dos grupos el $37 \%$ de las personas emplearon esta fuente de financiación en la última atención en salud. Los grupos más desfavorecidos en términos de aseguramiento fueron los que agrupan a personas entre 15 y 30 años, esto probablemente este relacionado con las condiciones de desempleo y subempleo en nuestro país, que afecta principalmente a personas jóvenes y que representa una barrera para el aseguramiento que está ligado al empleo formal.

En los análisis efectuados por estado conyugal, el ser soltero(a) representa una menor probabilidad de acceder a los servicios de consulta médica en los dos períodos, en el 2000, estar en unión libre también representa una desventaja en este sentido. En los dos años de estudio ser casado(a) representa mayor oportunidad de acceder a los servicios. Con respecto al estado conyugal y financiamiento el $21 \%$ de las personas casadas emplearon los recursos propios como fuente de financiación frente a 36 \% de las personas viviendo en unión libre y separadas y al $34 \%$ de las solteras. En cuanto al aseguramiento, cerca de la mitad de las personas solteras, en unión libre y separadas no tenían afiliación al SGSSS según 
la ENH del 2000 este comportamiento se explica por las características del aseguramiento que permiten a una persona afiliarse gracias a su relación conyugal de casado(a) en calidad de beneficiario, para las personas solteras y separadas no existe esta posibilidad.

En este análisis se encontró que en el 2000 el acceso a los servicios de salud estuvo más ligado al ingreso, pero en las dos encuestas las diferencias en acceso entre los quintiles de ingreso fueron significativas. Los resultados muestran diferencias en el acceso a los servicios que se consideran inequidades en contra de las personas con menores ingresos. Se podría suponer una relación entre estas inequidades y características del sistema como la falta de cobertura universal y la presencia de copagos y cuotas moderadoras que se han constituido en barreras de acceso adicionales a las ya inherentes a la condición de pobreza de muchos hogares. La identificación específica de estas relaciones requiere la aplicación de instrumentos diseñados con este propósito. A partir de estos resultados se sugiere que las inequidades por niveles de ingreso se mantienen y que continúan siendo las personas de los quintiles más bajos, que a su vez son las personas con más necesidades, las que tienen menos oportunidades de acceder a los servicios, pues la probabilidad de consultar es mayor para el quintil de mayores ingresos y menor para los quintiles uno y dos.

En 1994 las personas del quintil superior manifestaron en mayor proporción tener derecho a utilizar los servicios de salud de una entidad específica, situación que disminuía a medida que descendía el nivel de ingreso, aunque en este caso hay que tener en cuenta que para este momento (antes de implementada la reforma) la red de hospitales y centros de salud públicos con los mecanismos de subsidio a la oferta vigentes representaban la opción de atención más viable para la mayoría de población pobre. Siete años después de implementada la reforma los hallazgos mostraron que la posibilidad de no estar afiliado(a) al régimen contributivo del SGSSS es mayor para el quintil dos, seguido por el uno y que la afiliación representa un beneficio para los quintiles de mayor ingreso.

Respecto a la financiación de los servicios de salud que constituye uno de los componentes fundamentales de la evaluación de la equidad en un sistema de salud, los resultados muestran que las personas pertenecientes a los quintiles uno y dos son quienes en mayor proporción emplean los recursos propios o familiares como fuente de financiación de los servicios. (Figura 1). Teniendo en cuenta que el gasto de bolsillo es una fuente muy regresiva se diría que utilización de los servicios de salud podría estar contribuyendo al empobrecimiento de los hogares de menores ingresos. Considerando que el $46,5 \%$ de las mujeres pertenece a los 
dos primeros quintiles de ingreso (comparado con 34,9\% de los hombres), son ellas las más afectadas por los efectos regresivos de esta fuente de financiamiento que podría contribuir a acentuar las diferencias entre sexos en el nivel de ingreso. En el quintil de mayores ingresos las personas emplean en una menor proporción los pagos de bolsillo como fuente de financiación y tienen mayores posibilidades de acceder a los servicios, en este quintil se encuentra el $16 \%$ de las mujeres y el $24 \%$ de los hombres.

\section{DISCUSIÓN}

Los resultados obtenidos en la identificación de inequidades en la financiación coinciden con los hallazgos de Castaño y cols (14) quienes encontraron, al utilizar como variable de comparación los ingresos, un aumento en la regresividad de los pagos de bolsillo entre 1985 y 1997 e identificaron una tendencia hacia un impacto más regresivo de los mismos a partir de 1994. En este estudio el índice de concentración de los pagos de bolsillo comparados por nivel de ingresos evidenció la presencia de inequidades a favor de los más ricos. En otro estudio elaborado por los mismos autores (15) no encontraron diferencias en la probabilidad de utilizar un servicio de salud al comparar dos períodos: 1993 y 1997; adicionalmente encontraron que la probabilidad de utilizar los servicios por quintiles de ingreso no varió al comparar los dos años. Esta situación estaría indicando que en el período comprendido entre 1997 y 2000 tampoco se materializaron mejoras en materia de equidad por grupos de ingreso en el acceso a los servicios.

Figura 1. Recursos propios (\%) como fuente de financiación en la última atención en salud, por quintiles de ingreso. Año 2000

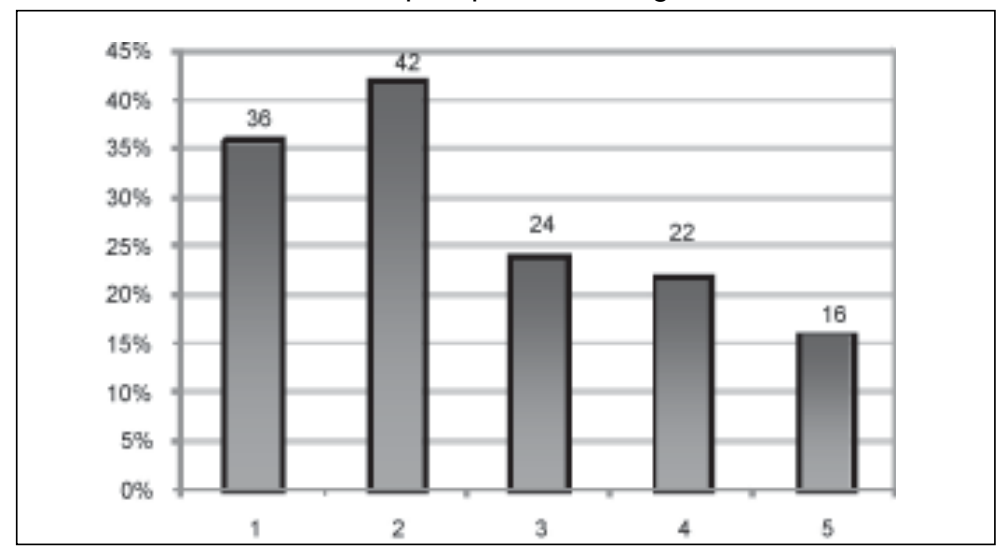


Céspedes y cols (16) concluyen que el actual sistema ha contribuido a eliminar las barreras económicas de acceso a los servicios (entendido como afiliación) beneficiando a los más pobres. Encontraron aumento en la afiliación que pasó del $23 \%$ al $57 \%$ y en el aseguramiento de los hogares pertenecientes al primer decil de ingresos que aumentó de 3,1 \% en 1993 a 43 \% en 1997. Frente a estos resultados es importante aclarar que el aseguramiento no equivale al acceso y que la comparación en estos términos presenta serias dificultades pues en el año 1993 el sistema de salud no estaba basado sobre una lógica de aseguramiento. Los indicadores de concentración en la utilización de los servicios de salud -componente del acceso real- no mostraron en este mismo estudio variaciones importantes aún después de ajustar por edad, género y necesidades.

En cuanto a los diferenciales en la necesidad sentida o percibida, los resultados del componente cualitativo de la investigación llevada a cabo por el grupo del Instituto FES (13) podrán ayudar a aclarar las causas ligadas al género que explican las diferencias encontradas en el presente estudio. Los investigadores evidenciaron que los hombres tienen dificultades para percibir necesidades de salud y tienen una percepción de la enfermedad como fracaso, mientras en las mujeres la percepción de enfermedad se corresponde con un problema que se puede resolver. Las mujeres tuvieron una actitud positiva hacia la consulta, los médicos y el sistema de salud, los hombres en cambio tuvieron actitud «reacia» frente a los mismos factores, argumentaron falta de tiempo para realizar las gestiones de salud y afirmaron consultar solo cuando el problema de salud es grave, las mujeres por el contrario expresaron que el trabajo doméstico o remunerado sí les dejaba tiempo para las gestiones de salud y se perciben a sí mismas como cuidadoras de la salud de su familia.

El presente estudio tiene limitaciones metodológicas derivadas de las características de las encuestas empleadas que no son estrictamente comparables por las preguntas que se formulan y las opciones de respuesta que se presentan. En este estudio no fue posible identificar las diferencias en el acceso a los servicios por regímenes de afiliación; lo cual merece un trabajo posterior de investigación. Este estudio no logró comparar los resultados obtenidos para cada encuesta por medio de un indicador de equidad lo cual brindaría información más precisa de los cambios presentados entre dos períodos, por lo tanto se sugiere para próximos estudios trabajar sobre instrumentos que permitan hacer análisis más precisos y emplear los indicadores de equidad descritos en la literatura.

Los resultados de este estudio permiten concluir que la reforma del sistema de salud colombiano no ha cumplido con su objetivo de garantizar equidad en el 
acceso y financiación de los servicios de salud. Las diferencias encontradas en acceso y financiación se consideran inequidades pues responden a variables de sexo, edad, estado conyugal e ingreso.

Algunas de las inequidades no presentan cambios en el período de estudio, mientras otras surgieron después de 1994, lo cual sugiere que existen características estructurales del sistema que han perpetuado las inequidades existentes y han hecho que aparezcan nuevas inequidades

\section{REFERENCIAS}

1. Almeida C. Reforma del sector salud y equidad en América Latina y el Caribe: conceptos, agenda, modelos y algunos resultados de implementación. Rev Ger Pol Salud. 2005; 4(9): 6-60.

2. Garay LJ, Rodríguez A. Colombia: Diálogo pendiente. Documentos de política pública para la paz. Bogotá: Planeta Paz., 2005

3. Hernández A, Vega R. El sistema colombiano de seguridad social en salud: desigualdad y exclusión. Rev Ger Pol Salud 2001; 1(1):48-73.

4. Whitehead M. The Concepts and principles of equity and health. World Health Organization. Regional Office for Europe. Copenhague; 1990. p.7

5. Whitehead M. The Concepts and principles of equity and health. Int J Health Serv 1992; 22(3): 429445.

6. De Haan A. Exclusión social en la política e investigación: operacionalización del concepto. En: Pobreza y Desigualdad: reflexiones conceptuales y de medición. Consuelo Corredor Editora. Universidad Nacional de Colombia, CINEP, Colciencias, GTZ. Bogotá; 1999. p. 22

7. Travassos C, Viacava F, Fernandes C, Almeida C. Desigualdades geográficas e sociais na utilização dos serviços de saúde no Brasil. Cien Saude Colet 2000; 5:133-150.

8. Casas-Zamora J, Gwatkin D. Las muchas dimensiones de la equidad en salud. Rev Panam Salud Publica 2002; 11(5/6).

9. Castaño R, Arbeláez J, Giedión U, Morales L. Evolución de la Equidad en el Sistema Colombiano de Salud. Naciones Unidas. CEPAL. Unidad de estudios especiales. Secretaría Ejecutiva. Serie: Financiamiento del Desarrollo No 108. Santiago de Chile; 2001. p. 10

10. Gómez E. Equidad, género y salud: retos para la acción. Rev Panam Salud Pública 2002; 11(5/ 6):454-461:i-ii.

11. Gómez E. Género, equidad y acceso a los servicios de salud: una aproximación empírica. Rev Panam Salud Publica 2002; 11(5/6):327-334

12. Weisman CS. Women's health care. Baltimore: The Johns Hopkins University Press; 1998.

13. Sarmiento L, Vargas CH. El Trabajo de las Mujeres en Colombia. Ed. Viva la Ciudadanía. Bogotá 2002

14. Castaño R, Arbelaez J, Giedion U, Morales L. Equitable financing, out of pocket payments and the role of health care reform in Colombia. Health Policy Plan 2002; 17 (Suppl 1): 5-11.

15. Castaño R, Arbelaez J, Giedion U, Morales L. Evolución de la Equidad en el Sistema Colombiano de Salud. Santiago de Chile: CEPAL; 2001.

16. Céspedes J, Jaramillo I, Martínez R. Efectos de la reforma de la seguridad social en salud en Colombia sobre la equidad en el acceso y la utilización de servicios de salud. Rev Salud Pública, 2000; 2(2): 145-164, 